\title{
An Abstract Transcript Notation for Analyzing Interactional Construction of Meaning in Online Learning
}

\author{
Daniel Suthers, Nathan Dwyer, Ravi Vatrapu and Richard Medina \\ Laboratory for Interactive Learning Technologies, Dept. of Information and Computer Sciences \\ University of Hawai $i$ \\ collaborative-representations@hawaii.edu
}

\begin{abstract}
This work is based on the premise that the interactional construction of meaning is as important in online settings as it is face-to-face, especially in collaborative learning. Yet most studies of online learning use quantitative methods that assign meaning to contributions in isolation and aggregate over many sessions, obscuring the situated procedures by which participants accomplish learning through the affordances of online media. Methods for studying the interactional construction of meaning are available, but have largely been developed for brief episodes of face-to-face data, and need to be adapted to online learning where media resources, time scale, and synchronicity differ. In order to resolve this tradeoff, we have prototyped an abstract transcript notation to support sequential and interactional analysis of distributed and asynchronous interactions. The paper describes applications to data derived from asynchronous interaction of dyads and small groups.
\end{abstract}

\section{Introduction}

Online learning is becoming increasingly prevalent and important in both formal and informal science education [1], being implemented under a variety of "blended" learning models [20] and K-12 settings [21] as well as strictly online models in university education [19]. Online collaborative learning brings together social processes of learning and representational aids for this learning, providing a fertile area for research and development while serving an important application. An understanding of how participants appropriate and are influenced by the affordances of the medium is needed to adequately inform the design of the learning experience and the resources that support it. Because learning is largely social $[9,36,37]$, it is also critical to understand the entwinement of individual trajectories of participation [33].

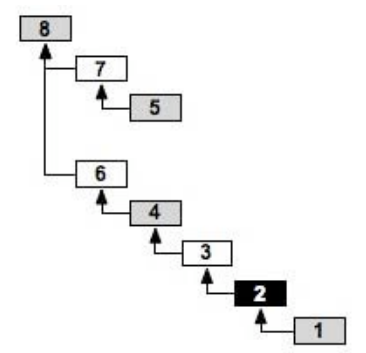

Figure 1. Reply structure of a threaded
discussion

Most studies of online learning assign meaning to contributions in isolation, obscuring the interactionally constructed meaning. These methods also aggregate over many sessions, losing the actual procedures by which participants accomplish learning through the affordances of online media [18]. Methods for studying the interactional construction of meaning are available $[12,16]$, but have largely been developed for brief episodes of face-to-face data, and require adaptation to online environments where media resources, time scale, and synchronicity all differ. Analyses that are too closely tied to media representations may fail to identify interactional sources of coherence. As a simple example, consider the reply structure from a threaded discussion shown in figure 1. There appears to be two divergent lines of discussion, but an analysis to be presented in this paper shows that this is not the case. Additionally, since most research on online learning is currently conducted in text-based tools, we lack methods to study how richer representations might mediate online learning. The analytic tradeoff between scalability and fidelity must be resolved in order to inform the design of improved online learning environments and activities that engage participants more deeply in intersubjective meaning-making during collaborative inquiry. The immediate objective of the 
work reported in this paper is to resolve this tradeoff by scaling up sequential and interactional analysis to distributed and asynchronous interactions while remaining grounded in participants' use of media. The long term objective is to obtain a deep understanding of how learning is accomplished in technologymediated settings by analyzing computer-mediated interactions that span long durations of time and take place in different media among groups of various sizes. As a first step, we have developed an abstract transcript notation, the "dependency graph," that provides a media-independent foundation for analyzing how participants build on each others' contributions. This notation was initially prototyped for synchronous computer-mediated communication (CMC) as reported in [32]. The present paper reports on improvements motivated by applications to analysis of asynchronous interaction of dyads and small groups.

The remainder of this paper briefly introduces some theoretical assumptions behind the work; motivates and describes the dependency graph as the basis for analysis of meaning-making in online settings; and provides examples of this analysis to two sources of data (a laboratory study of asynchronously interacting dyads, and an online course discussion).

\section{Learning and Meaning-Making}

This work makes an important assumption concerning how learning takes place in social settings. Learning is conceived of not merely the transfer of information but rather as an interactional process of change. This conception is compatible with theories of learning that identify socially embedded individuals $[9,36]$, social systems [10] or communities [37] as the locus of change. Although we focus on learning in social settings, an interactional view of learning is also consistent with research on "individual" learning, such as reading [4] and solving physics problems [6]. The change we call "learning" need not be deliberately sought: it is a result of participants' attempts to make sense of a situation [8]. Meaning-making, as we will call it in this paper, takes place at multiple levels: solving a problem, maintaining interpersonal relationships, and/or affirming identity in a community [5]. We maintain that to study learning in social settings we must necessarily study the practices of intersubjective meaning-making: how people in groups make sense of situations and of each other [33]. Meaning is interactionally constructed and situated: the meaning of a given contribution is best understood as a function of its relationships to prior interactions and indexically with respect to the physical and social context. (We use the term "contribution" in its colloquial sense of actions offered to advance a joint endeavor, specifically including actions in shared media that express attitudes and attentional orientation as well as information. A discussion of how asynchronous interaction problematizes contribution theory's distinction between "utterance" and "contribution" [7] is outside the scope of this paper.) Meaning-making is mediated by the physical and social environment in diverse ways [10, 15, 37]. As designers of media for online learning, this mediation gives us an avenue for influencing meaning-making and possibly learning through the socio-technical affordances of the tools that we design [23].

\section{Analysis of Online Learning}

The properties of online media pose special challenges for analysis. This section considers how the major analysis paradigms handle the analysis of meaningmaking that takes place via online media, and offers an alternative.

\subsection{Quantitative Methodologies}

Many empirical studies of online learning follow a quantitative paradigm in which contributions (such as discussion postings) are annotated under some coding system (e.g., [25]) and then statistical methods are used to compare counts across groups to draw conclusions concerning aggregate (average) group behavior. Such methods are suitable for testing hypotheses concerning certain types of differences between groups, and indeed we have used them for this purpose in our own work [34]. Yet, "coding and counting" cannot capture the in situ practices of intersubjective meaning making, and hence the most essential part of collaborative learning is missed. There are two basic problems. First, the meaning of an act is assigned to it as an isolated unit, missing the sequential construction of this meaning. Second, when data is aggregated, one loses the situated methods by which knowledge construction is accomplished in a given situation and medium. Without observing how media affordances are used in particular learning accomplishments (or how opportunities are lost), it may be more difficult to generate design recommendations.

\subsection{Qualitative/Sequential Methodologies}

Methodologies that find the meaning and significance of each act in the context of prior interaction are available, and include Conversation Analysis [26], Interaction Analysis [16], Narrative Analysis [13], and the family of analysis methods loosely classified as "sequential analysis" [27]. Many of these approaches (especially the first two cited) draw upon the ethnomethodological assertion that order emerges from the participants' interaction [11]. Typically, video or transcripts of naturally occurring 
interactions are studied to uncover the methods by which participants make themselves accountable to each other and accomplish their objectives. For examples applied to the analysis of learning, see [2, $17,18,24]$. This paradigm is becoming increasingly important in computer supported collaborative learning because an approach that focuses on accomplishment through action is necessary to truly understand the role of technology affordances [30].

Yet, these methods also have limitations, mostly due to assumptions about the interactional properties of the media they study. Both Conversation Analysis and Interaction Analysis are concerned with face-to-face interaction. Production blocking and the ephemerality of spoken interactions constrain communication in such a manner that turns [26] and adjacency pairs [28] are appropriate units of analysis for face-to-face data. These units of analysis are not as appropriate for CMC since most online media support simultaneous production and persistence of contributions. Contributions may become available to other participants in unpredictable orders, may not be immediately available, and because of the medium's persistence participants may at any time address earlier contributions [14]. Conceptual coherence is decoupled from temporal adjacency. It follows that we cannot simply focus analysis on the relationships between adjacent events. Nor can we treat $\mathrm{CMC}$ as a degenerate form of face-to-face interaction (e.g., by seeking an analog to adjacency pairs) since people adapt to these media attributes and use them to create new forms of interaction [3, 14].

Some methods tie their analytic notations closely to data representations that mirror the media within which interaction takes place. For example, analysis methods based on annotation of units (e.g., categorizing utterances, or tagging video time points) will not support relational analysis; and methods that rely on fully linearized representations of data will not capture the asynchonicity of CMC. The spatial distribution of contributions across media or workspaces should also be considered. We seek an alternative representation of the data that abstracts from the particular media of interaction while retaining links to the original data format, supporting analysis of and comparisons across heterogeneous media.

\subsection{An Alternative Methodology}

Based on considerations discussed in the last two sections, we sought an analytic structure that maintains the sequential and situational context of activity so that an account of the interactional construction of meaning is possible, does not assume that the medium of interaction has any particular interactional properties (e.g., synchronicity, availability of contributions, or persistence), but records these properties where they exist.

The properties of asynchronous online media require a unit for analysis of interaction that accommodates relevance between noncontiguous contributions and allows for tracking of availability as a prerequisite to awareness and access. Additionally, this unit of analysis must be applicable to the wide variety of temporal, spatial, and social scales of online activities. Since collaborative learning is only possible when something is shared and transformed between participants, we chose the concept of uptake for this unit of analysis. Uptake is the event of a participant doing something with previously expressed information, attitudes and attentional orientation or other reifications of prior participation [32]. Uptake affirms and transforms the taken-up by interpreting it as having certain relevance for further participation. A participant can take up one's own prior reifications as well as those of others: by identifying both, analysts can characterize the mixture of intrasubjective and intersubjective knowledge construction. Uptake is similar to the "thematic connections" of Resnick, et al. [22], but allows for media as well as linguistic relationships. Uptake must sometimes be inferred. We found it useful to separate the relatively objective evidence of participants' media actions from the analysts' identification of uptake. This led to the development of the "dependency graph."

\subsubsection{Dependency Graph}

An act of uptake is simultaneously the expression of a conception and the interpretation of prior expressions: the act of taking up and the conception resulting from that act are mutually constitutive. However, to help simplify the task of analysis, the methodology and its associated data structure-the dependency graph-make a distinction between evidence for the existence of a conception-represented as a vertex in the graph-and the dependency of this evidence on the evidence for the existence of other conceptions - represented as arcs in the graph. We speak of dependencies between evidence for conceptions rather than dependencies between conceptions so that the first phase of the analysis can proceed without having to identify the conceptions. We leave identification of the dependencies of conceptions on other conceptions to the next phase of interpretation (section 3.3.2), and believe that at this level the unity of conceptions and uptake can no longer be avoided.

The dependency graph is a directed acyclic graph in which each vertex represents evidence for the existence of a conception and edges represent dependencies between this evidence and other vertices. It is a hypergraph: a vertex can depend on multiple other 
vertices. See [32] for further development of the graphtheoretic definition that provides the basis for computational support of the construction and examination of dependency graphs.

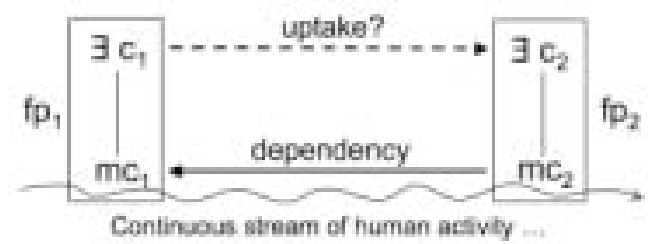

Figure 2. Schema for a dependency

A schema for a basic dependency is shown in figure 2. The existences of conceptions (e.g., $c_{1}$ and $c_{2}$ in figure 2) are evidenced by media coordinations (e.g., $\mathrm{mc}_{1}$ and $\mathrm{mc}_{2}$ in figure 2). This definition is motivated by an implication of the theory of distributed cognition [15]. According to Hutchins, a system that consists of one or more persons and artifacts relies on coordinations of conceptual and perceptual representations. Hereinafter we will use representation to refer to perceptual representations. An intentional media coordination implies a conception. We include any media manipulation for which a conceptual counterpart is possible, including not just creation and editing of utterances, messages, or objects in a workspace, but also (for example) deliberate access to media objects, grouping objects in space, gestures, and expressions of attitude and selective attention.

We call the vertices of the graph fixed points (e.g., $\mathrm{fp}_{1}$ and $\mathrm{fp}_{2}$ in figure 2) because they are instances of analytical stability in the continuous stream of human activity that provide "points of departure" for the analysis. Fixed points are analytic entities that index to the data, and are not to be understood as substituting for the data itself. Fixed points (e.g., fp and $\mathrm{fp}_{2}$ ) constitute claims that conceptions (e.g., $\mathrm{c}_{1}$ and $\mathrm{c}_{2}$ ) exist as evidenced by the indexed data (e.g., $\mathrm{mc}_{1}$ and $\mathrm{mc}_{2}$ ). They are not points in the data: they may index to a range or region of the data.

The arcs of the dependency graph (e.g., from $\mathrm{fp}_{2}$ to $\mathrm{fp}_{1}$ ) indicate dependencies of fixed points on prior fixed points that provide evidence for potential uptake events. The fact that evidence for one conception $c_{2}$ depends on evidence for another conception $c_{1}$ suggests that $c_{2}$ itself may depend on $c_{1}$, i.e., that there is uptake. Dependences range from media dependencies (e.g., direct manipulation of or reference to the media representations indexed by prior fixed points) to lexical and conceptual dependencies (e.g., re-expression of ideas in language).

\subsubsection{Interpreting the Dependency Graph}

A dependency graph is not a complete analysis, but rather is an intermediate structure that can be thought of as an abstract transcript that indexes to the original data. The analysis itself identifies sequences of dependencies between fixed points as (inverted) sequences of uptakes, and interprets the significance of the sequence and the nature of the uptake based on the theoretical phenomena of interest, such as argumentation, knowledge construction, or intersubjective meaning-making. Because the construction of evidence and the analytic interpretation are separated, the dependency graph can serve as a basis for comparison and integration of multiple theoretical interpretations, i.e., serve as a boundary object [31] for the study of collaboration.

\section{Examples}

In this section we provide two examples from exploratory analyses conducted over the past year. The first example is based on data from dyads interacting in a laboratory setting. This example illustrates analysis of intersubjective meaning-making in an asynchronous context where detailed data is available. The second example is based on server logs of asynchronous threaded discussions in an online course. This example illustrates how our method can be adopted to conventional online learning settings, and the advantage of an analysis that is not tied too closely to media structure.

\subsection{Dyads in a Laboratory Setting}

We developed our analytic methodology using existing data from a study that was designed to test hypotheses not directly relevant to the present paper [35]. Participants interacted via computers using a shared "evidence mapping" workspace to identify the cause of a disease on Guam (ALS-PD). Information was distributed across participants such that information sharing was necessary to refute weak hypotheses and construct a more complex hypothesis. The protocol for propagating updates between workspaces was asynchronous [35]. Rich data including server logs and video capture of the screens are available to us, so we are able to examine the interaction in great detail. Other data includes individual essays that participants wrote at the end of the session.

Our analysis treated the essay writing as continuing participation rather than as a single point of measurement. We sought to identify whether and how the construction of the essays was accountable to the prior session, and especially whether interaction between participants influenced the essays. For each session analyzed, we began with the particiapnts' essays and traced dependencies back into the session to identify uptake trajectories that may have influenced the essays. Some sessions were chosen for analysis 
because there was convergence in the content of the essays and we wanted to identify how this convergence was achieved interactionally. Other sessions were chosen to examine a failure to converge or to share vital information. In both cases we want to relate significant instances of intersubjective uptake or failure thereof to practices of media appropriation. The first example presented below is of the former type: both participants (referred to as P1 and P2) mentioned "duration of exposure" as a factor in their essays, and the analysis sought to identify how this convergence was accomplished.

4.1.1 Relationship of the dependency graph to the data. In this section we describe the relationship of the elements of a dependency graph to data, drawing on an analysis we conducted for one session. We constructed the dependency graph in Microsoft Visio ${ }^{\mathrm{TM}}$ based on inspection of software $\log$ files and of video of participants' screens. The dependency graph we constructed is large (over 180 fixed points and 220 dependencies) and cannot be presented in print media. A relevant subgraph is shown in figure 3: many fixed points and dependencies are omitted for simplicity. P1's media actions and fixed points for P1's conceptions are on the top and P2's actions and fixed points are on the bottom. In general, time flows left to right, but this being an asynchronous setting we cannot assume that a contribution is available as soon as it is created, nor can we assume that the clocks on each client were synchronized (they were not). The vertical lines in each participant's half demarcate when the local client updated that participant's workspace to display new work by the partner.

In figure 3 , the rounded boxes with text in them summarize data on which the presented portion of the graph is based, including media manipulation during the session and text written in the essay. The small square boxes represent fixed points, each of which claims the existence of a conception evidenced by media manipulations (such as editing the evidence map) or access to the partner's contribution (evidence map objects must be opened to be read). The links between media events and fixed points may be read as evidential links, showing how observed coordinations of representations provide evidence for the existence of conceptions, following [15].

Each fixed point is assigned a numerical identifier. Since the fixed point represents coordination between the representation and a conception, it is most accurate to discriminate between the fixed point, the media event that provides evidence for the fixed point, and the conception implied by the coordination represented by the fixed point. We will use these specific terms whre they are warranted, but generally we will use the numeric identifier as shorthand to refer to the media event and the conception as well as the fixed point that binds them together.

Arrows between the fixed points represent dependencies (potential uptake relations). Dotted arrows represent intrasubjective and solid arrows represent intersubjective dependencies. An intersubjective dependency is always evidenced by perception of some media entity. The conception evidenced by the perception is dependent on but not necessarily identical to the conception evidenced by the creation of the media entity. For example, the conception claimed by fixed-point $20 \mathrm{a}$ is evidenced by access to the same media object the creation of which evidenced the existence of the conception claimed by fixed point 20. P1's conception (20a) upon reading this note at 1:50:23 is dependent on but not necessarily identical to P2's conception (20) when creating this note at 1:41:40.

Dependencies can also be evidenced by editing media objects or by lexical similarity, and can be further evidenced by temporal and spatial proximity. For example, at 1:52:06, P1 added a comment (expressing conception 10) to the same note object that she had just read at 1:50:23. (A note object can contain a sequence of comments from both participants.) Since the expression of the conception 10 could not have taken place unless this media object existed, we have evidence that conception 10 depends on (and therefore takes up) conception 20a. The same example illustrates lexical and temporal evidence for a dependency. The media coordination that evidences 10 uses the phrase "environmental factors," which is present in the note that was accessed at 1:50:23, providing further evidence that 10 is dependent on 20a. Finally, the media coordination event that evidences conception 10 takes place 103 seconds after the media coordination event that evidences conception 20a, providing circumstantial evidence by temporal proximity that 10 depends on 20a. 


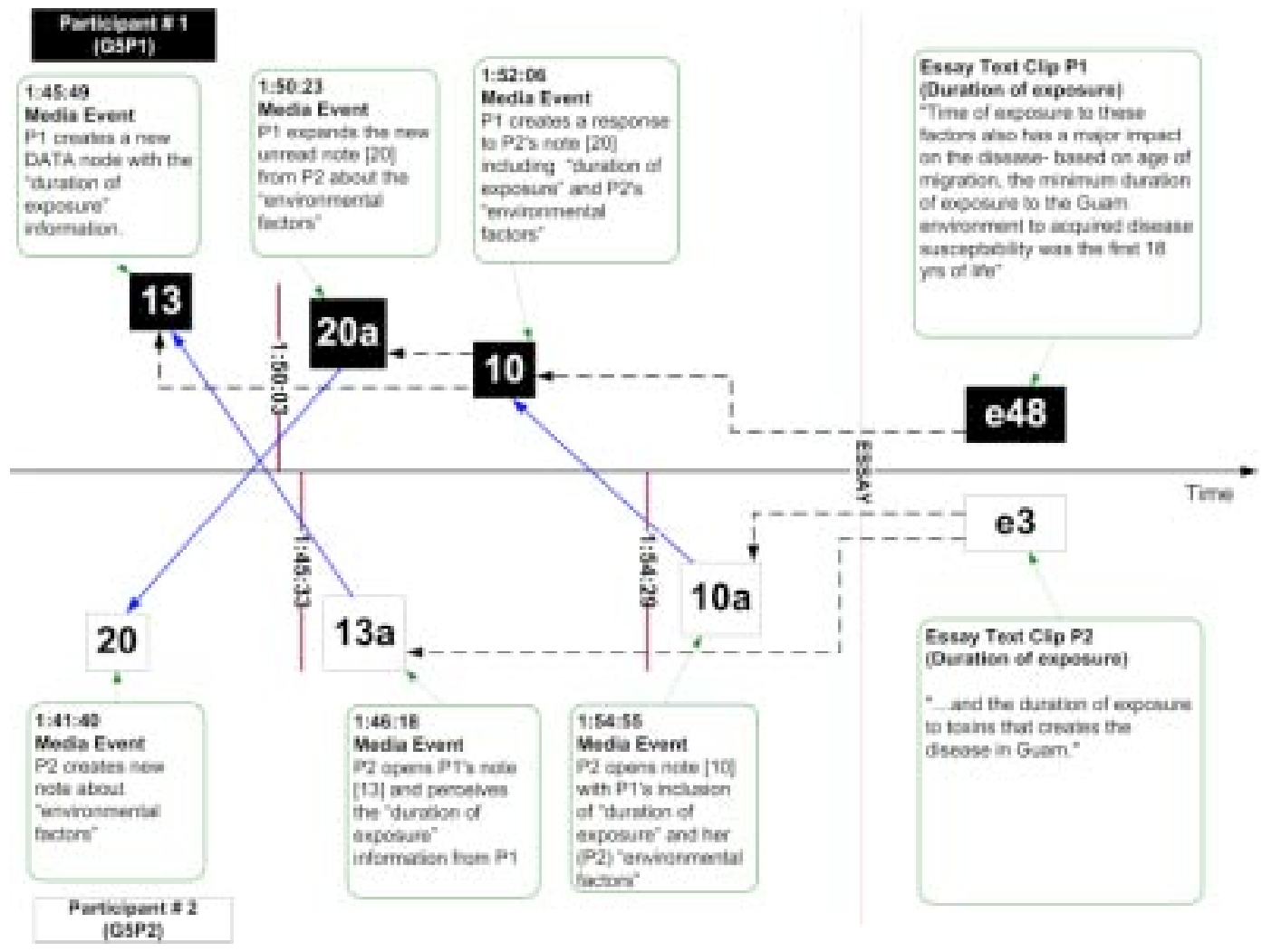

Figure 3: Fragment of a dependency graph for asynchronous dyads

4.1.2. Interpretation of the dependency graph. In this section we walk through the graph of figure 3 to trace out a simple example of the interactional construction of meaning while highlighting further features of the graph. At 1:41:40, P2 creates a note summarizing environmental factors as disease causes (fixed-point 20). This note is not yet visible to P1. Shortly after that in clock time but asynchronously from the participants' perspectives, P1 creates a data object (13) concerning the minimum exposure to the Guam environment needed to acquire the disease. Subsequently, a workspace refresh (1:50:03) makes the note expressing conception 20 available to P1. Fixedpoint 20a represents the conception that results from P1's access to this note at 1:50:23, and the corresponding arrow to 20 represents evidence for intersubjective uptake. Later at 1:52:06, P1 adds a comment to the note object (10). We interpret this as an uptake of the conceptions of $20 \mathrm{a}$ and 13 , as evidenced by the media-level facts for $20 \mathrm{a}$ discussed in the previous section and the incorporation of the concept of "duration of exposure" from 13. Clearly, 10 is an integrative contribution.

Awareness of representational elements is not symmetrical in asynchronous media. At one point in the interaction just described, both 13 and 20 existed but neither was available to the other participant.
Analysis must account for the contents of both workspaces to address these kinds of issues. The vertical line notation indicates when the media manipulations of other participants become available to a given participant, but analysis cannot simply rely on the appearance of a media object in a workspace. The analysis must find evidence that a contribution was actually accessed, which is why we need the "perceptual" fixed points such as 20a. Notations developed for face-to-face and synchronous communication often assume a single context and immediate availability of contributions. These are reasonable assumptions for those media but significantly limit those notations' applicability to asynchronous media.

Let us now examine how information originally available only to P1 (13) and P1's integration of it (10) become available to P2. Sometime after 13 was expressed, a refresh (1:45:33) makes the corresponding data object available to $\mathrm{P} 2$, who accesses it as 13a. Subsequently (after P2 does other work not shown), another refresh (1:54:29) makes 10 available to P2, soon accessed at 1:54:55 (10a). Since P2 has considered both 13a ("duration of exposure") and P1's endorsement of the relevance of duration of exposure for environmental factors (10a), we view P2's inclusion of these concepts as "the duration of 
exposure to toxins" in her essay (e3) to be an uptake of both of these conceptions. P1's essay portion (e48) also evidences uptake of the environmental factors $(20 \mathrm{a}$ via 10). The "round trip" from 20 through 20a, 10 and back to $10 \mathrm{a}$ and e3 represents intersubjective meaningmaking on a small scale. We cannot rule out that e3 is uptake of only 13-13a and hence a one-way transfer of information, but nor can we rule out that P1's endorsement of the importance of the idea in 10, taken up in 10a, also influenced P2's inclusion of this idea in the essay. It is plausible that both were a factor.

\subsection{An Asynchronous Online Discussion}

The laboratory setting provided far richer instrumentation than is typical in online learning applications. In order to explore how our method can be adopted to conventional online learning settings, we analyzed server $\operatorname{logs}$ of asynchronous threaded discussions in an online graduate course on collaborative technologies. The collaborative learning software (discourse.ics.hawaii.edu, developed in our laboratory) records message-opening events as well as message postings, but there is no other record of participants' manipulations of the screen.

Figure 4 provides a fragment of the dependency graph we constructed in one analysis. After reading a paper on socio-constructivist, socio-cultural, and shared cognition theories of collaborative learning, a student facilitator suggested that students write "grant proposals" to evaluate learning in the course itself, and discuss how their choice of theory would affect how they approach the evaluation. The episode took place over 5 days, indicated in figure 4 by vertical lines for midnight of each day. The actual reply structure is shown as thick grey arrows between the postings (rounded boxes). This reply structure is also summarized in figure 1, replicated in the lower left of figure 4 . The episode of figure 4 was chosen because it illustrates conceptual integration across two subthreads, and hence the independence of conceptual dependencies from media structure. The small graph lower left is sufficient for analysis of the threading structure, but the main graph situates the threading structure in a richer network of conceptual dependencies and temporal relationships.

Stepping through the graph, at 8 the instructor $(\mathrm{P} 2)$ has posted a comment concerning a prior contribution that used the phrase "socio-cultural" but seemed to express a socio-cognitive approach. Unfortunately, the assignment mentioned "socio-constructivist" rather than "socio-cognitive" and the student $(\mathrm{P} 1)$ reading this message (8a) is confused by the different name. She raises questions about the distinction in two separate replies, 7 and 6 . (Not shown in this simplified graph is a sequence of message reads between 7 and 6: P1 appeared to be searching for more information on the topic.) The next day, P2 returns, sees 7 (7a), replies with an explanation of "socio-cultural" in 5, and then starts down the other subthread. Seeing 6 (6a) the source of the confusion becomes apparent and P2 replies with a terminological clarification (4). Later that day, $\mathrm{P} 1$ reads both threads $(5 \mathrm{a}, 4 \mathrm{a})$ but replies only to the second with a "thank you" (3). On the third day, $\mathrm{P} 3$ reads this entire exchange in both threads $(7 \mathrm{~b}$, $5 \mathrm{~b}, 6 \mathrm{~b}, 4 \mathrm{~b}, 3 \mathrm{a})$ and replies to the last "thank you" message with a meta-comment about the confusion.

Participants' reading and posting strategies as well as the default display state and no-edit policy of the medium affect whether conversations are split up or reintegrated. By posting two separate replies (rather than editing her first reply-not allowed-or responding to that reply), P1 opens up the possibility of a divergent discussion. By following a strategy of reading and replying to each message one at a time, P2 continues the split that $\mathrm{P} 1$ has started. The discussion tool also allows one to scroll through a single display of all messages that one has opened in a single discussion. By following a strategy of reading all messages before replying, P3 brings these separate subthreads together. However, the reply structure of the discussion tool does not allow this convergence to be expressed in the medium: P3 must reply to one of the messages, so replies to the last one she read. Her message seems odd as a reply to the "thank you," as it is referring to "several of our grant proposals." In a sequence of reads not shown, P3 had read through the grant proposals about an hour before posting 2 .

The dependency graph captures aspects of the coherence of the mediated interaction that are not apparent in the interface itself (e.g., the threaded reply structure in this example). Although some of this coherence can be recovered through analysis of quoting practices [3], our analysis goes further to include (for example) lexical and temporal evidence for coherence, evidence that can also be partially automated. This ability to identify trajectories that are independent of yet influenced by media structures is an important strength of the method. 


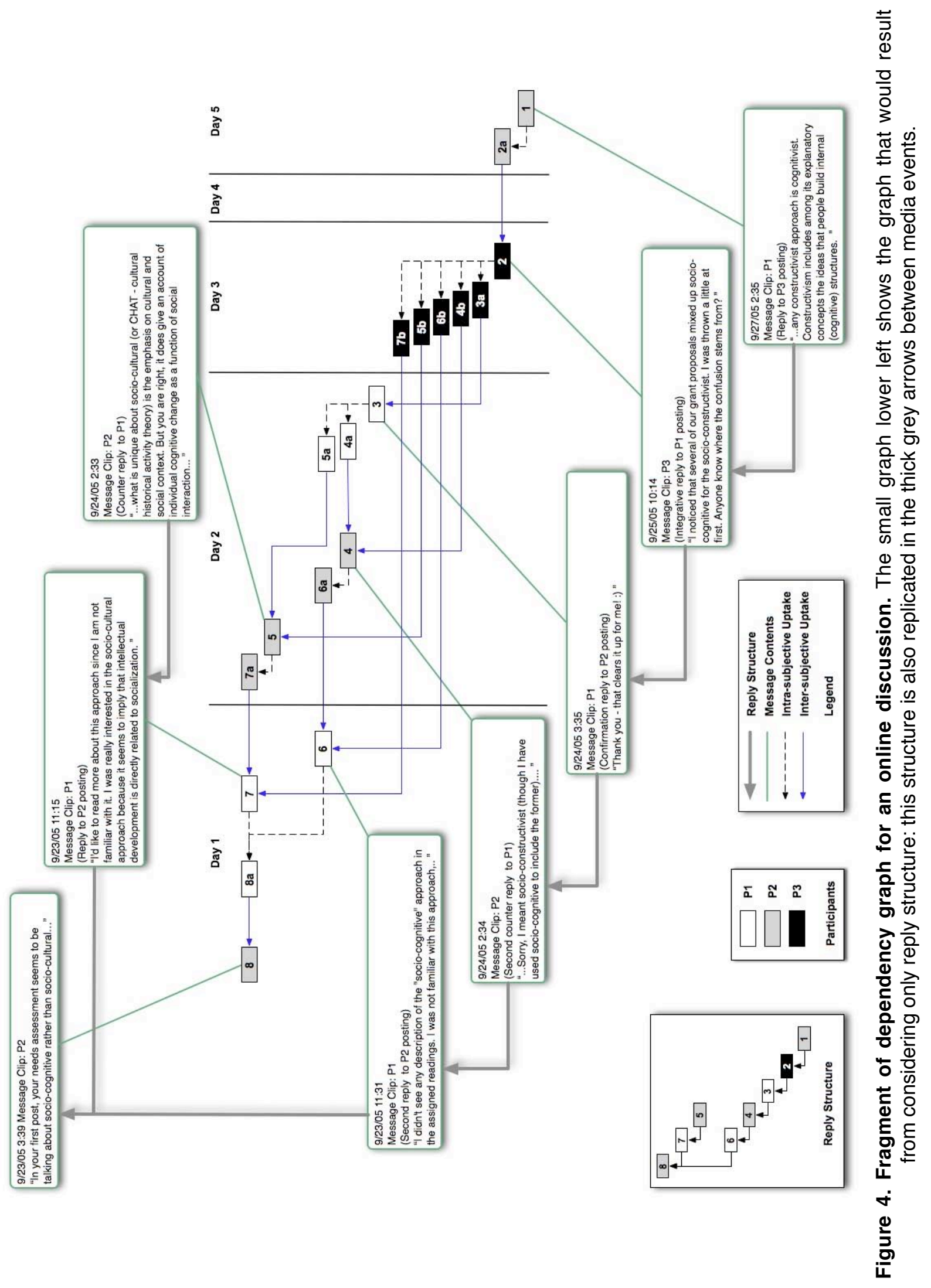




\section{Summary and Discussion}

This paper presents an abstract transcript notation, the dependency graph, which provides the foundation for scaling up the advantages of sequential and interactional analysis to longer term distributed and asynchronous interactions. The approach has been prototyped on data derived from synchronous [32] and asynchronous interaction of dyads and small groups. In each of the analyses we conducted, we were able to identify an interaction episode showing the potential of the method for producing a feature-rich analytical artifact supporting multilayered interpretation.

The dependency graph is media-agnostic. It is a record of the multiple coordinations that took place in an interaction and maps out their interdependencies. However, it is not media ignorant; it can bring in medium-specific information. Meaning-making can be identified independently of the media but is linked to media by the fixed points, so the relationship between meaning-making and the media can be examined.

The dependency graph enables one to separate out individual trajectories and identify when contributions are available to and accessed by each individual; to examine how these trajectories affect each other; and to step back and analyze the composite web of interpretations. "Group cognition" [29] is observable as the result of multiple individuals allowing their individual actions to be influenced by the perception and interpretation of other's behavior.

There are presently several disadvantages of the methodology. The major disadvantage is that it is time consuming to construct a dependency graph. We developed the full graph (180 fixed points and 220 dependencies) for the first example over a period of about 8 months during which we also engaged in intensive weekly discussions to refine the methodology and associated theory. More recently, we have conducted similar analyses of other sessions in several days. Customized software support can help address this problem by partially automating data collection and the construction of the graph through media, lexical and temporal dependencies. The present work develops the representational specifications for such a tool. Once we are sure that these specifications are correct we will develop the tool. A related problem is the difficulty of retrieving information from and obtaining selective views of the dependency graph. Software support will also address this problem by displaying the uptake graph at multiple granularities and through filters, compressing it in time and/or scanning for patterns. An analyst need not even use a graph representation at all: visualization tools can convert the underlying graph model into whatever visualization is useful.

In interpreting our graphs we have encountered two other issues related to the intrinsic incompleteness of the graph as a data representation. One must be careful not to make inferences based on the absence of fixed points and dependencies in the graph: any graph is partial and can be extended indefinitely due to the continuous nature of human action. One must not conduct an analysis entirely by using the dependency graph. In addition to being a structure of interest in its own right, the graph should be used as an index to the original media records. Visualization software can help address this problem by overlaying or simultaneously displaying the graph with the source media.

In ongoing work, we continue to apply the methodology to a diversity of data in preparation for development of software support tools. Our objective is to speed up the analysis of intersubjective meaningmaking to the point where it need not be considered only a tedious variation on micro-analysis, but can also be efficiently applied on a larger scale.

\section{Acknowledgments}

This work was supported by the National Science Foundation under award 0093505. Any opinions, findings, and conclusions or recommendations expressed in this paper are those of the authors and do not necessarily reflect the views of the National Science Foundation.

\section{References}

[1] I. E. Allen and J. Seaman, Growing by Degrees: Online Education in the United States, 2005, The Sloan Consortium (2005), pp. http://www.sloanc.org/resources/growing_by_degrees.pdf.

[2] M. Baker, Computer-mediated argumentative interactions for the co-elaboration of scientific learning tasks., in Andriessen, Baker and Suthers, eds., Arguing to Learn: Confronting Cognitions in Computer-Supported Collaborative Learning Environments., Kluwer, Dordrecht, 2003, pp. 47-78.

[3] F. Barcellini, F. Détienne, J.-M. Burkhardt and W. Sack, Thematic coherence and quotation practices in OSS design-oriented online discussions, Proceedings of the 2005 International ACM SIGGROUP Conference on Supporting Group Work (GROUP '05), ACM Press, Sanibel Island, Florida, USA, 2005, pp. 177-186.

[4] I. L. Beck, Questioning the Author: An Approach for Enhancing Student Engagement with Text, International Reading Association, Delaware, 1997.

[5] J. P. Bronckart, Theories of action, speech, natural language, and discourse., in J. V. Wertsch, P. D. Rio and A. Alvarez, eds., Sociocultural Studies of Mind, Cambridge University Press, New York, 1995, pp. 7591.

[6] M. Chi, M. Bassok, M. Lewis, P. Reimann and R. Glaser, Self-Explanations: How Students Study and Use Examples in Learning to Solve Problems, Cognitive Science, 13 (1989), pp. 145-182. 
[7] H. H. Clark and S. E. Brennan, Grounding in communication, in L. B. Resnick, J. M. Levine and S. D. Teasley, eds., Perspectives on Socially Shared Cognition, American Psychological Association, 1991, pp. 127-149.

[8] B. Dervin, Sense-Making's Journey from Metatheory to Methodology to Method: An Example Using Information Seeking and Use as Research Focus, in B. Dervin and L. Foreman-Wenet, eds., Sense-Making Methodology Reader: Selected Writings of Brenda Dervin, Hampton Press, Inc., Cresskill, New Jersey, 2003, pp. 133-163.

[9] W. Doise and G. Mugny, The Social Development of the Intellect, International Series in Experimental Scoial Pscychology, Pergamon Press, 1984.

[10] Y. Engestrom, Expansive Learning at Work: toward an activity theoretical reconceptualization, Journal of Education and Work, 14 (2001).

[11] H. Garfinkel, Studies in Ethnomethodology, PrenticeHall, Englewood Cliffs, New Jersey, 1967.

[12] C. Goodwin and J. Heritage, Conversation Analysis, Annual Review of Anthropology, 19 (1990), pp. 283307.

[13] D. Hermann, Narrative Theory and the Cognitive Sciences, Center for the Study of Language and Information., Stanford, 2003.

[14] S. C. Herring, Interactional coherence in CMC, Journal of Computer Mediated Communication, 4 (1999).

[15] E. Hutchins, Cognition in the Wild, The MIT Press, Cambridge, Massachusets, 1995.

[16] B. Jordan and A. Henderson, Interaction Analysis: Foundations and practice, The Journal of the Learning Sciences, 4 (1995), pp. 39-103.

[17] T. Koschmann and C. LeBaron, Reconsidering common ground: Examining Clark's contribution theory in the $O R$, in $\mathrm{K}$. Kuuttii, E. Karsten, G. Fitzpatrick, P. Dourish and K. Schmidt, eds., ECSCW 2003: Eighth European Conference on ComputerSupported Collaborative Work, Kluwer Academic Publishing, Amsterdam, 2003.

[18] T. Koschmann, A. Zemel, M. Conlee-Stevens, N. Young, J. Robbs and A. Barnhart, How do people learn: Member's methods and communicative mediation, in R. Bromme, F. W. Hesse and H. Spada, eds., Barriers and Biases in Computer-Mediated Knowledge Communication, Kluwer Academic Press, Amsterdam, 2005, pp. 265-294.

[19] F. Mayadas, Asynchronous Learning Networks: A Sloan Foundation Perspective, Journal of Asynchronous Learning Networks, 1 (1997), pp. http://www.aln.org/alnweb/journal/jaln_issue1.htm\# mayadas.

[20] M. Orey, V. J. McClendon and R. M. Branch, eds., Educational Media and Technology Yearbook, Libraries Unlimited, Westport, CT, 2006.

[21] P. L. Parker, Learning Technologies and their Impact on Science Education: Delivering the Promise, Australian Science Teachers Journal, 46 (2000), pp. 9-20.

[22] L. B. Resnick, M. Salmon, C. M. Zeitz, S. H. Wathen and M. Holowchak, Reasoning in conversation, Cognition and Instruction, 11 (1993), pp. 347-364.
[23] P. Resnick, Beyond Bowling Together: SocioTechnical Capital, in J. M. Carroll, ed., HumanComputer Interaction in the New Millennium, ACM Press, Upper Saddle River, NJ, 2002, pp. 647-672.

[24] J. Roschelle, Designing for cognitive communication: Epistemic fidelity or mediating collaborating inquiry, in D. L. Day and D. K. Kovacs, eds., Computers, Communication \& Mental Models, Taylor \& Francis, London, 1996, pp. 13-25.

[25] L. Rourke, T. Anderson, D. R. Garrison and W. Archer, Methodological issues in the content analysis of computer conference transcripts., International Journal of Artificial Intelligence in Education, 12 (2001).

[26] H. Sacks, E. A. Schegloff and G. Jefferson, A simplest systematics for the organization of turn-taking for conversation, Language, 50 (1974), pp. 696-735.

[27] P. Sanderson and C. Fisher, Exploratory sequential data analysis: Foundations, Human-Computer Interaction, 9 (1994), pp. 251-318.

[28] E. A. Schegloff and H. Sacks, Opening up closings, Semiotica, 8 (1973), pp. 289-327.

[29] G. Stahl, Group Cognition: Computer Support for Collaborative Knowledge Building, MIT Press, Cambridge, MA, 2006.

[30] G. Stahl, T. Koschmann and D. D. Suthers, CSCL: An historical perspective., in R. K. Sawyer, ed., Cambridge Handbook of the Learning Sciences, Cambridge University Press, Cambridge, UK 2006.

[31] S. L. Star, The structure of ill-structured solutions: Boundary objects and heterogeneous distributed problem solving, in L.Gasser and M. N. Huhns, eds., Distributed Artificial Intelligence, Morgan Kaufmann, San Francisco, 1990, pp. 37-54.

[32] D. D. Suthers, A qualitative analysis of collaborative knowledge construction through shared representations Research and Practice in Technology Enhanced Learning 1(2006), pp. 1-28.

[33] D. D. Suthers, Technology affordances for intersubjective meaning-making: A research agenda for CSCL, International Journal of Computers Supported Collaborative Learning, 1 (2006), pp. (in press).

[34] D. D. Suthers and C. Hundhausen, An experimental study of the effects of representational guidance on collaborative learning, Journal of the Learning Sciences, 12 (2003), pp. 183-219.

[35] D. D. Suthers, R. Vatrapu, S. Joseph, N. Dwyer and R. Medina, Representational effects in asynchronous collaboration: A research paradigm and initial analysis, Proceedings of the 39th Annual Hawai'i International Conference on System Sciences, IEEE Computer Society Press, Poipu, Hawai'i, 2006, pp. CD-ROM.

[36] L. S. Vygotsky, Mind in society, Harvard University Press, Cambridge, MA, 1978.

[37] E. Wenger, Communities of Practice: Learning, Meaning and Identity, Cambridge University Press, Cambridge, 1998. 